\title{
Behavior of streamwise rib vortices in a three-dimensional mixing layer
}

\author{
By J. M. Lopez ${ }^{1}$ AND C. J. Bulbeck ${ }^{1}$
}

The structure and behavior of a streamwise rib vortex in a direct numerical simulation of a time-developing three-dimensional incompressible plane mixing layer is examined. Where the rib vortex is being stretched, the vorticity vector is primarily directed in the vortex axial direction and the radial and azimuthal velocity distribution is similar to that of a Burger's vortex. In the region where the vortex stretching is negative, there is a change in the local topology of the vortex. The axial flow is decelerated and a negative azimuthal component of vorticity is induced. These features are characteristic of vortex breakdown. The temporal evolution of the rib vortex is similar to the evolution of an axisymmetric vortex in the early stages of vortex breakdown. The effect of vortex breakdown on other parts of the flow is, however, not as significant as the interaction between the rib vortex and other vortices.

\section{Introduction}

In the main, the term vortex breakdown has been associated with large scale flows. In aeronautics, vortices generated by swept leading edges and the trailing vortices generated at wing tips are observed to undergo vortex breakdown. In such flows, vortex breakdown is characterized by the sudden deceleration of the axial flow, with an associated sudden expansion of the core. Downstream, the flow is unsteady and seemingly disorganized. With the exception of a few idealized flows designed to study vortex breakdown in isolation, our knowledge of vortex breakdown is based solely on such large scale flows.

Recently, there have been suggestions that vortex breakdown occurs naturally over a much wider range of flows and scales than has previously been expected. One example is the breakdown of the side vortices associated with a jet in cross-flow (Kelso 1991). Orszag (1991) has suggested that vortex breakdown may be present in the results of computations of homogeneous isotropic turbulence reported by She, Jackson and Orszag (1990). He observed that coherent structures in homogeneous isotropic turbulence (which are tube-like vortical structures recently referred to by the term 'worms') have characteristic flow features suggestive of vortex breakdown. He observed that the tangent of the helix angle (ratio of azimuthal to axial component) of the velocity vector is larger than that of the vorticity vector. This represents one of the necessary conditions for vortex breakdown developed by Brown

1 Defence Science and Technology Organisation, Air Operations Division - Aeronautical Research Laboratory, Melbourne, Australia 


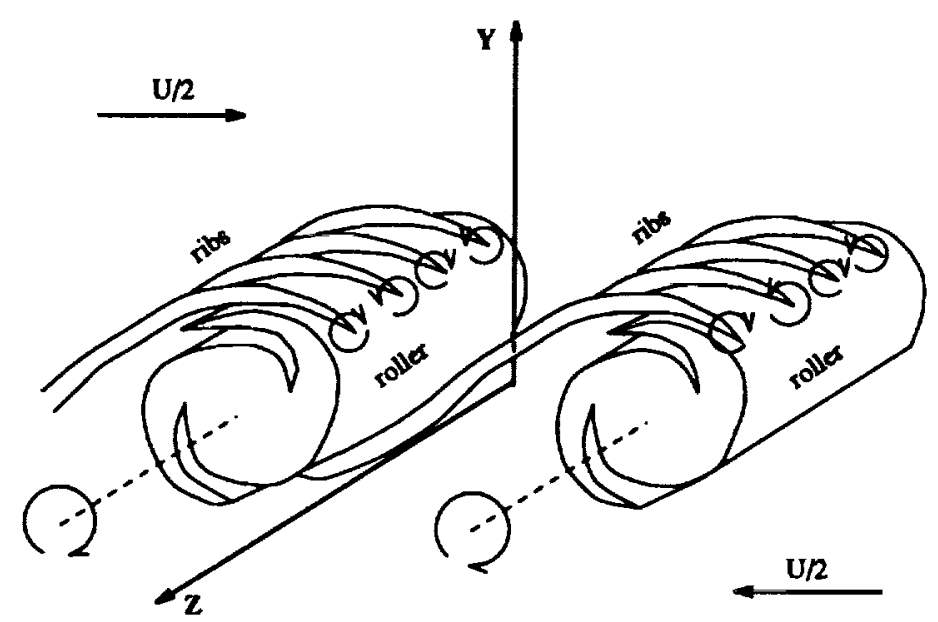

Figure 1. Diagram of the structures in a plane mixing layer.

and Lopez (1990) on the basis of steady, axisymmetric swirling flow theory. Chen et al. (1990) have also suggested that vortex breakdown may occur within the threedimensional plane mixing layer flow computed by Moser and Rogers (1992). It is this latter suggestion that is the subject of investigation in this study.

The flow computed by Moser and Rogers (1992) is a three-dimensional, incompressible plane mixing layer formed between two streams moving at differing velocities. In such a plane mixing layer, spanwise vortices, called 'rollers', are generated by the Kelvin-Helmholtz instability of the layer. These rollers undergo 'pairing' whereby neighboring rollers co-rotate and amalgamate. Three-dimensional instability of the mixing layer also gives rise to counter-rotating 'rib' vortices which exist in the region between the rollers (the 'braid' region) and extend from the bottom of one roller to the top of the next (Figure 1). The vorticity in the rib vortices is predominantly perpendicular to that of the rollers. An observed change in the character of the local topology along a rib vortex, from stable focus/stretching to unstable focus/contracting, was taken by Chen et al. (1990) as evidence for vortex breakdown. We wish to determine whether the phenomenon of vortex breakdown is indeed responsible for the observed change in local topology.

The approach used in the present study to identify vortex breakdown is to project flow field quantities in the vicinity of the rib vortex onto a locally cylindrical polar co-ordinate system. The cylindrical polar co-ordinate system is constructed such that the axial direction corresponds to the axis of the rib vortex. Local topology (explained in detail in section 2), axial velocity, azimuthal vorticity, and enstrophy are all examined in this co-ordinate system. Comparisons are made with axisymmetric vortex breakdown in confined swirling flows as computed by Brown and Lopez (1990). Vortex-vortex interactions are also investigated to determine whether they 
or the vortex breakdown are responsible for the observed spiralling of the rib vortex core.

The motivation behind the present research is twofold. Firstly, vortex breakdown can make a significant contribution to mixing. If it occurs in a flow such as the plane mixing layer, which is undergoing a transition to turbulence, mixing associated with vortex breakdown may provide a mechanism for transition. Secondly, if vortex breakdown can occur in a coherent structure in the mixing layer flow, it may also be occurring in coherent structures in other flows, such as in the worms found in homogenous isotropic turbulence.

\section{Global and local classiflcations of flow field topologies}

Complex flow fields can be interpreted by classifying their topology. This can either be done globally or locally. In the global approach, developed by Perry and Fairlie (1974) and extended by Chong, Perry, and Cantwell (1990), the critical points of the flow field are identified. The critical points in a flow field are those points where all three velocity components are simultaneously zero relative to a global observer. A local Taylor series expansion of the velocity field with respect to space co-ordinates is made at each of these critical points, and the invariants of the resulting $3 \times 3$ Jacobian matrix, the velocity gradient tensor $A$, are used to completely classify the topology of this critical point.

In the local approach, the co-ordinate system translates without rotation while following the fluid particle. That is, each point in the flow field is considered to be a critical point since the velocity of each point is zero relative to a local observer. The topology of each point in the flow is then classified, as in the global approach, by considering the local velocity gradient tensor at each point in the flow. Due to the Galilean invariant nature of the velocity gradient tensor and hence any property based on this tensor, the local topological classification of each fluid flow point is independent of the observer. This local classification of the flow field was first used by Chen et al. (1990) and is the approach used in this study.

The velocity gradient tensor can be decomposed into its symmetric and antisymmetric parts, i.e.

$$
A_{i j}=\frac{\partial u_{j}}{\partial x_{i}}=S_{i j}+W_{i j}
$$

where

$$
S_{i j}=\left(\frac{\partial u_{i}}{\partial x_{j}}+\frac{\partial u_{j}}{\partial x_{i}}\right) / 2
$$

is the rate-of-strain tensor and

$$
W_{i j}=\left(\frac{\partial u_{i}}{\partial x_{j}}-\frac{\partial u_{j}}{\partial x_{i}}\right) / 2
$$

is the rotation tensor. $A_{i j}, S_{i j}$ and $W_{i j}$ are all tensors of second order.

For a second order tensor, $\mathbf{A}$, where $\lambda_{1}, \lambda_{2}$, and $\lambda_{3}$ are the eigenvalues and $e_{1}$, $e_{2}$, and $e_{3}$ are the eigenvectors, then

$$
(\mathbf{A}-\lambda \mathbf{I}) e=\mathbf{0},
$$


and the corresponding characteristic equation

$$
\operatorname{det}[\mathbf{A}-\lambda \mathbf{I}]=0
$$

may be written as

$$
\lambda^{3}+P \lambda^{2}+Q \lambda+R=0 .
$$

The invariants $P, Q$, and $R$ are

$$
\begin{gathered}
P=-S_{i i} \\
Q=\left(P^{2}-S_{i j} S_{j i}-W_{i j} W_{j i}\right) / 2 \\
R=\left(-P^{3}+3 P Q-S_{i j} S_{j k} S_{k i}-3 W_{i j} W_{j k} S_{k i}\right) / 3 .
\end{gathered}
$$

For an incompressible flow as is being considered here, $P=0$, and the topology of the flow is completely classified by $Q$ and $R$.

The characteristic equation $\lambda^{3}+Q \lambda+R=0$ can have (i) all real distinct roots, (ii) all real roots with at least two equal, or (iii) one real root and a conjugate pair of complex roots. The curve $27 R^{2}+4 Q^{3}=0$ separates the regions of real and complex roots. Chong, Perry and Cantwell (1990) define regions where the velocity gradient tensor has complex eigenvalues as vortex cores, i.e. regions where $Q>-3(R / 2)^{2 / 3}$ are vortical in nature. The eigenvalues of the velocity gradient tensor determine the local kinematics of the flow, and these are determined by the invariants $Q$ and $R$. If $Q>-3(R / 2)^{2 / 3}$, then a conjugate pair of complex eigenvalues result, and hence the trajectories will be spiralling locally. Whether the spirals are stable or unstable is determined by the sign of the real eigenvalue, which in turn is determined by the sign of $R$. If $R>0$, then the spiral is unstable, and to conserve mass, the local topology is of the unstable focus/contracting (UFC) type. For $R<0$, it is of the stable focus/stretching (SFS) type. For the degenerate case of $R=0$, the trajectory is a closed loop rather than a spiral. For $Q<-3(R / 2)^{2 / 3}$, the local flow is strain dominated, and for $R<0$, the topology is of the stable node/saddle/saddle (SNSS) type, and for $R>0$, it is of the unstable node/saddle/saddle (UNSS) type.

\section{Determination of the rib vortex axis}

The following discussion centers on the flow case 'HIGH1P' of Moser and Rogers (1992) in which the initial conditions of the calculation involved a high-strength three-dimensional perturbation. The structure of the vortex was determined at non-dimensional time $t=28.5$, after a pairing of the main rollers had occurred. Chen et al. (1990) have suggested that vortex breakdown may be occurring at this time based on their observation of a subsequent change in the local topology along the vortex core.

In order to describe the structure of the vortex, its associated velocity and vorticity fields, a co-ordinate system that is peculiar to the vortex is required rather than one using the co-ordinate system in which the governing equations are cast. A local cylindrical polar co-ordinate system is chosen where the axial direction is tangent to the axis of the vortex. A difficulty arises in defining the axis of the vortex. Here, 


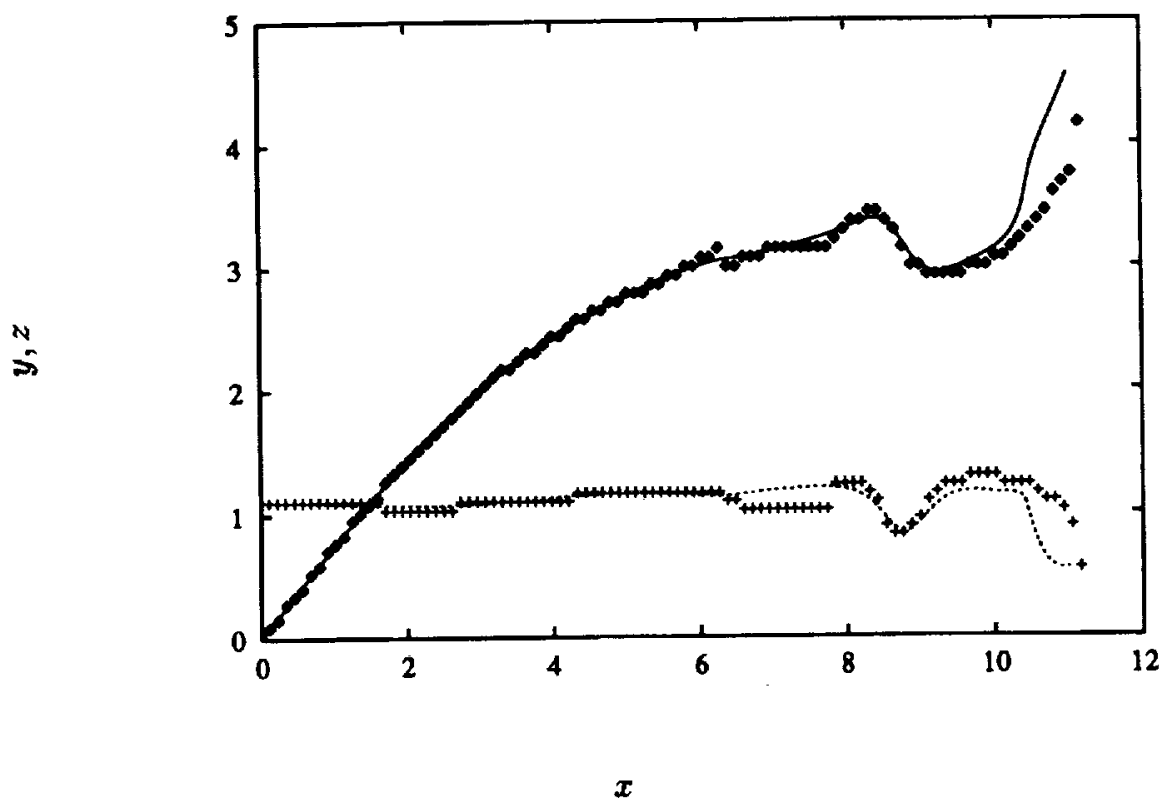

FigURE 2. Location of the vortex axis projected onto $x-y$ and $x-z$ planes at time $t=28.5$.

a distinction is made between the 'centerline' and the 'axis' of the vortex. If the modal decomposition of the azimuthal components of the vortex velocity and vorticity fields are dominated by even modes, then the centerline and the axis coincide. If, however, the odd modes dominate, then the centerline spirals around the vortex axis.

There has been much controversy over what constitutes the centerline of a vortex (e.g. Yates and Chapman 1992). In regions where the vortex is predominantly axisymmetric, its centerline can be reasonably approximated by either the loci of enstrophy local maxima, or a vortex line extending from a point of maximum enstrophy in the vortex core. The vortex core is taken to be the region enclosed by the maxima of the azimuthal component of velocity. The axis of the vortex has been determined by both methods and the location of the axis projected onto $x-y$ and $x-z$ planes are given for $t=28.5$ in Figure 2. There is very little difference in the determined axis from either method for $x<8$.

The structure of the rib vortex at $t=28.5$ can be described in terms of three distinct regions. Region I extends from the mid-braid plane to the axial location where the local topology changes from stable focus/stretching to unstable focus/contracting. The mid-braid plane is the $z-y$ plane mid way between two spanwise rollers (see Figure 1), and in the time-developing calculations of Moser and Rogers (1992), this plane is a symmetry plane. Region II extends downstream from the point of topology change. The structure of the vortex in regions I and II 
(a)

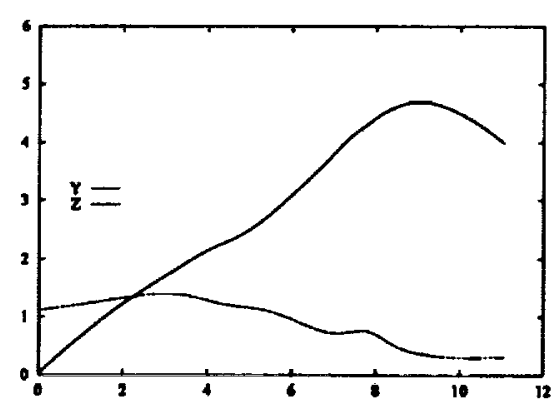

$\boldsymbol{s}$

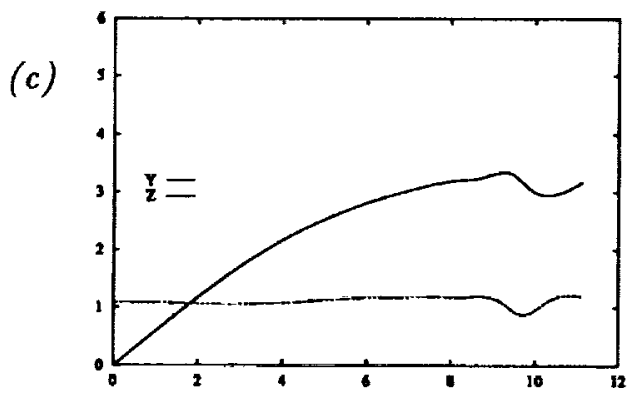

(b)

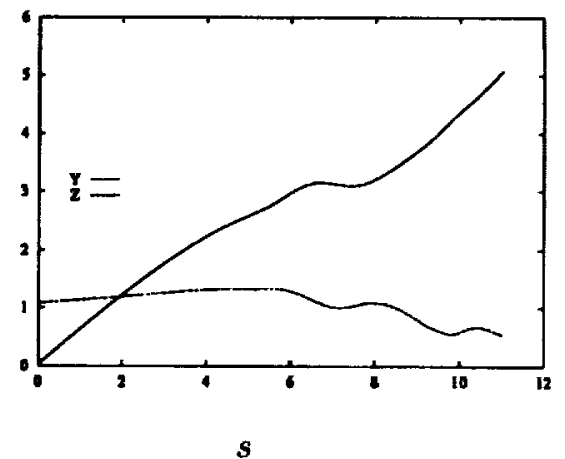

$\mathbf{S}$

Figure 3. Spanwise $Z$, and vertical $Y$, locations of the vortex axis in terms of distance along the axis at times $(a) t=22.3,(b) t=25.3$ and $(c) t=28.5$.

is dominated by the axisymmetric azimuthal mode. The rib vortex then evolves to a stage where it is no longer dominated by the axisymmetric mode. This region is denoted as region III. Region III does not appear at the earlier times considered since the centerline does not spiral about the axis at these times.

\section{Temporal and spatial evolution of the streamwise rib vortex}

The development of the rib vortex was investigated by analyzing the flow field at three non-dimensional times, $t=22.3, t=25.3$, and $t=28.5$ (where the first pairing occurs at $t=23.4$ ). At each time, the vortex axis was determined by the techniques outlined in section 3 . The vortex axes are shown in Figure 3 . The parameter $s$ is the distance along the vortex axis from the mid-braid plane. Local cylindrical polar co-ordinates were determined at each of the three times. At any point on the vortex axis, the axial direction ax is defined as being tangent to the vortex axis, positive downstream. The radial direction $\mathbf{r}$ is defined in terms of the axial direction ax and the vertical direction $y$ by $\mathbf{r}=\mathbf{a x} \times \mathbf{y}$. Figure 4 illustrates the local cylindrical polar co-ordinate system.

The distribution of azimuthal velocity across the vortex core at a streamwise location downstream of the mid-braid plane but upstream of the topology change 


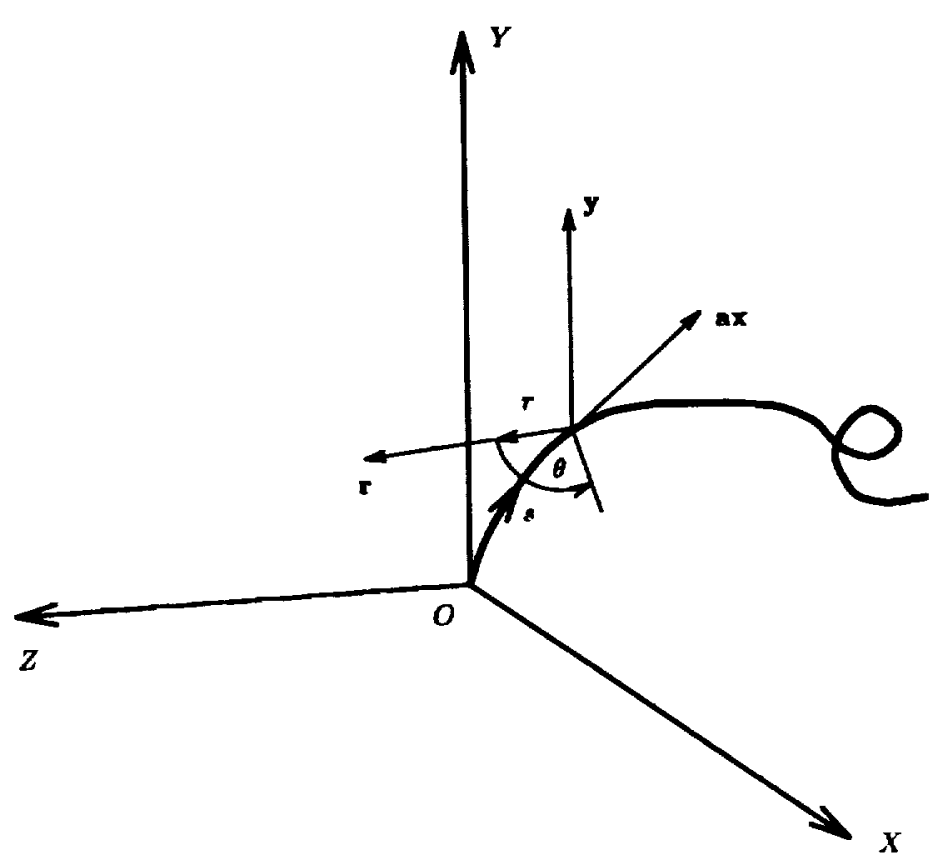

FiguRE 4. Local cylindrical polar co-ordinate system defined along the axis of the vortex.

$(s=1.0)$ at time $t=28.5$ is shown in Figure 5. The point $s=1.0$ lies in region I. Also shown is the azimuthal velocity for a Burger's vortex given by

$$
v_{\theta}(r)=\frac{\Gamma}{2 \pi} \frac{(1-\exp (-\alpha \gamma))}{\sqrt{2 \gamma}}
$$

where $\gamma=r^{2} / 2, \Gamma=2.2$ and $\alpha=18$. Both $\Gamma$ and $\alpha$ have been fitted to the data from the mixing layer calculation. Despite the highly three- dimensional nature of the flowfield, in region I the azimuthal velocity of the rib vortex is nearly axisymmetric and well described by the equation for a Burger's vortex.

Figure 6 shows the variation in the velocity gradient tensor invariants, $Q$ and $R$, along the vortex axis as well as the vortex stretching, $\omega_{i} S_{i j} \omega_{j}$, at the three times considered. The instantaneous exponential stretching rate for a vortex element is given by

$$
\zeta=\frac{d}{d t} \log \|\omega(t)\|
$$

where $\omega$ is the vorticity vector. In the inviscid limit (Dresselhaus and Tabor 1991), 


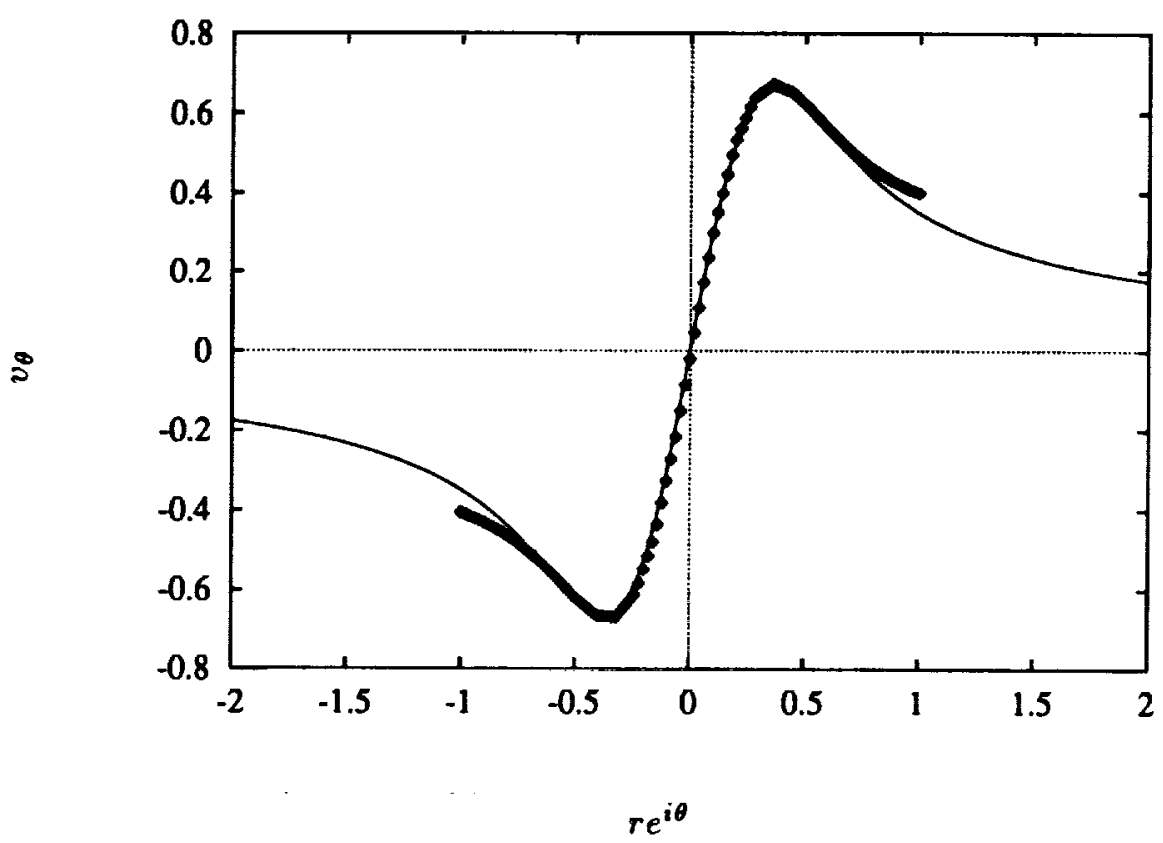

FIGURE 5. Radial distribution of azimuthal velocity at axial location $s=1.0$ and azimuthal locations $\theta=0$ and $\theta=\pi$, denoted by $\diamond$ and compared with a Burger's vortex azimuthal velocity distribution for $\Gamma=2.2$ and $\alpha=18$.

this may be expressed as

$$
\zeta=\sum_{i, j} S_{i j} \hat{\omega}_{i} \hat{\omega}_{j}
$$

where $\hat{\omega}$ is the unit vector in the direction of $\omega$. A related term, $\omega_{i} S_{i j} \omega_{j}$ is referred to as 'vortex stretching'. A vortex intensifies if it is being stretched, i.e. $\omega_{i} S_{i j} \omega_{j}$ is positive. The imposed axial strain will cause a decrease in the cross-sectional area of the vortex, and to conserve angular momentum, the angular velocity, and hence vorticity, will increase. There is a strong negative correlation between the vortex stretching and the third invariant $R$, i.e. when one is positive, the other is negative. Since the second invariant is positive in the vortex core region, positive vortex stretching corresponds to a local topology of the stable focus/stretching type and negative vortex stretching to the unstable focus/contracting type.

Figure 7 shows the radial variation of the local topology along the vortex axis at $\theta=0$ and $\pi$ at the three different times. The light regions correspond to a stable focus/stretching type topology and the dark regions to an unstable focus/contracting type topology. Along the centerline, the local topology changes from stable focus/stretching to unstable focus/contracting, and this denotes the boundary between regions I and II. This boundary moves downstream at a non-dimensional speed 0.37 . 
(a)

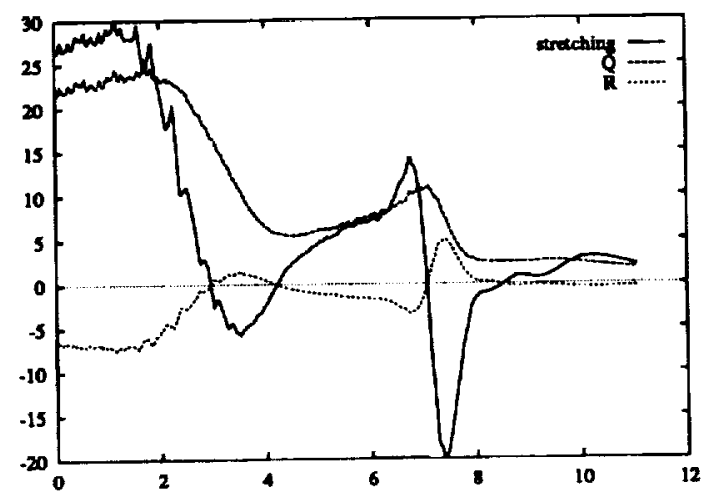

$\boldsymbol{s}$

(b)

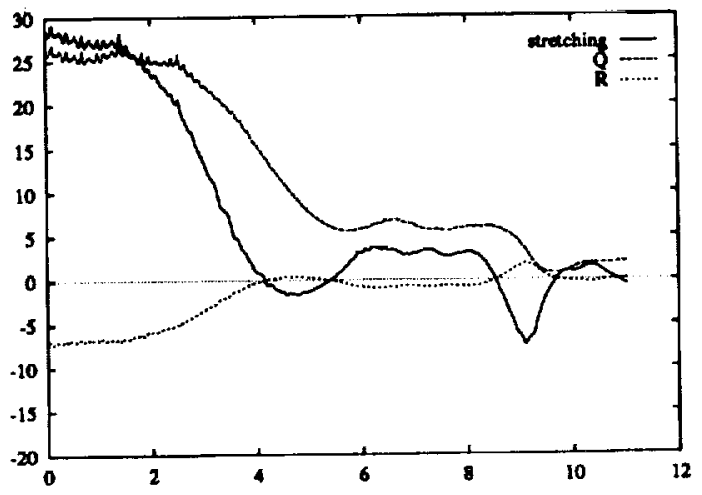

$\boldsymbol{s}$

(c)

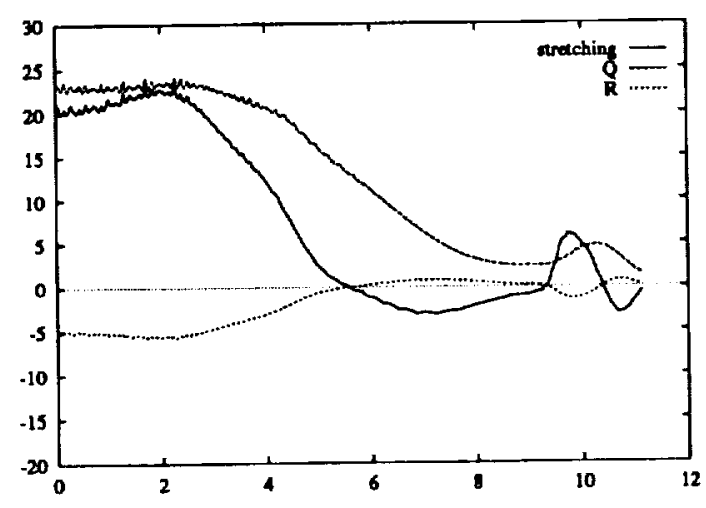

$\boldsymbol{S}$

FIGURE 6. Variation of the vortex stretching term, $\omega_{i} S_{i j} \omega_{j}$, and the second and third invariants of the velocity gradient tensor, $Q$ and $R$, with distance along the vortex axis $s$, at times (a) $t=22.3,(b) t=25.3$, and $(c) t=28.5$. 
(a)

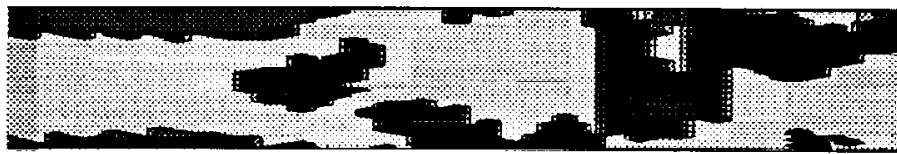

(b)

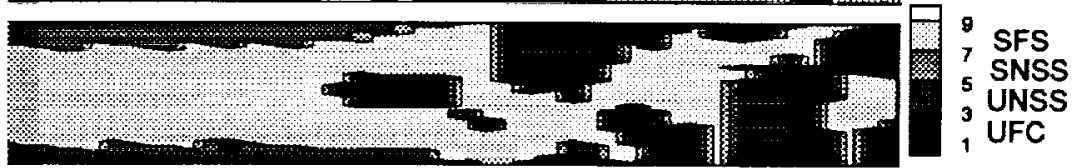

(c)

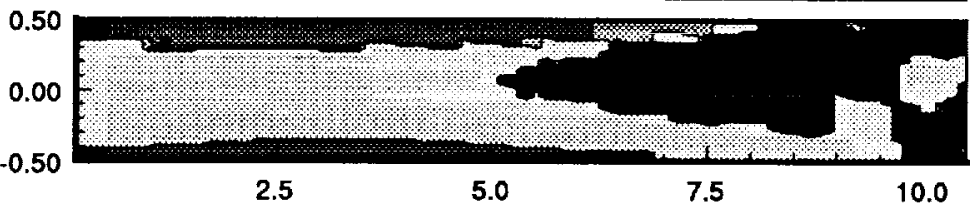

Figure 7. Local topology in the vortex core region of the streamwise rib vortex at times (a) $t=22.3$, (b) $t=25.3$ and $(c) t=28.5$.

(a)

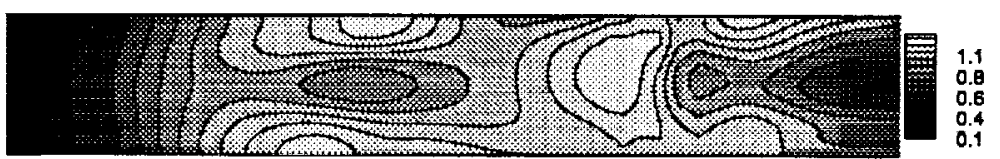

(b)
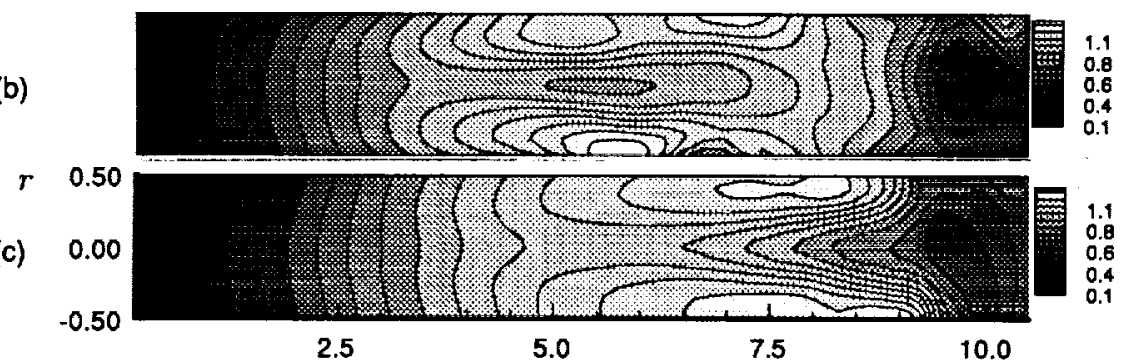

$s$

FIgURE 8. Axial velocity in the vortex core region of the streamwise rib vortex at times $(a) t=22.3$, (b) $t=25.3$ and $(c) t=28.5$.

Figure 8 shows contours of axial velocity. It may be seen that deceleration of the axial flow is associated with the topology change. Furthermore, the axial velocity in the region of the topology change is approximately equal to, or perhaps a little greater than, the speed at which the boundary between regions I and II moves downstream, indicating that the boundary is advected downstream by the local velocity. 
(a)

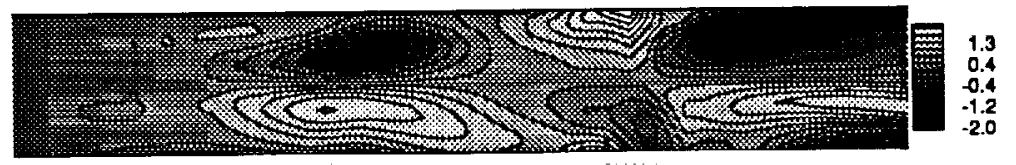

(b)

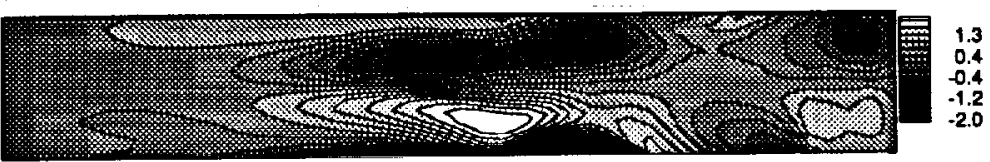

(c)

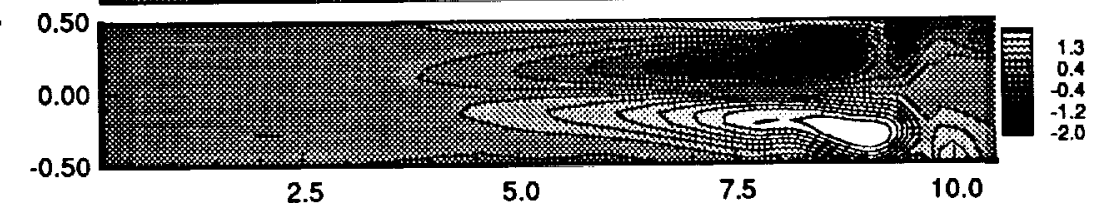

$s$

FIGURE 9. Azimuthal vorticity in the vortex core region of the streamwise rib vortex at times $(a) t=22.3,(b) t=25.3$ and $(c) t=28.5$.

(a)

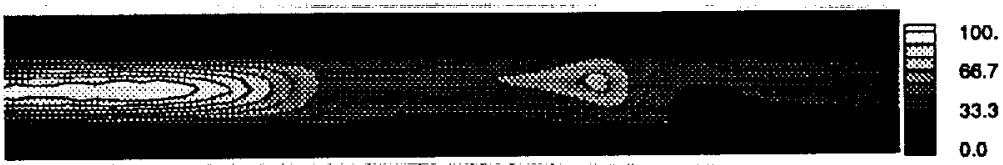

(b)

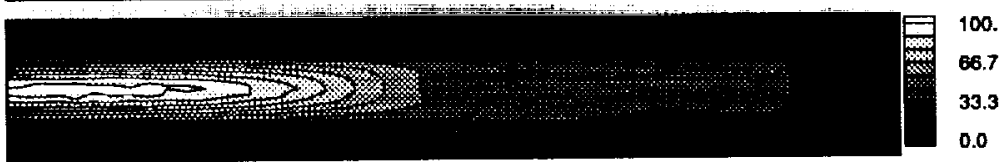

$r$

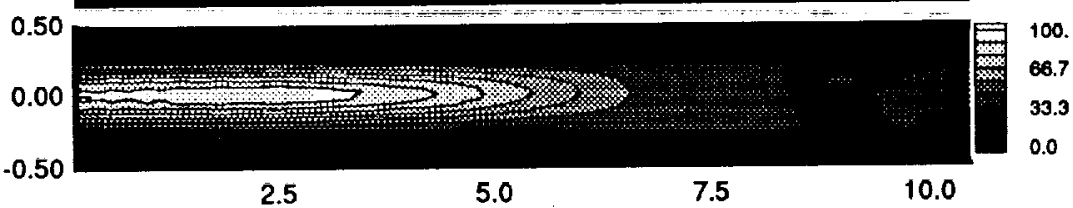

$S$

FIGURE 10. Enstrophy in the vortex core region of the streamwise rib vortex at times (a) $t=22.3,(b) t=25.3$ and $(c) t=28.5$.

In Figure 9, the distribution of the azimuthal component of vorticity is shown. In region $I$, for all three times, the azimuthal vorticity is nearly zero. The vorticity vector is almost entirely directed in the axial direction. Just upstream of region II, a negative component of azimuthal vorticity appears. It has a local extremum off the axis. This negative component of azimuthal vorticity off the axis induces a retardation of the axial flow. This scenario is entirely consistent with the early 


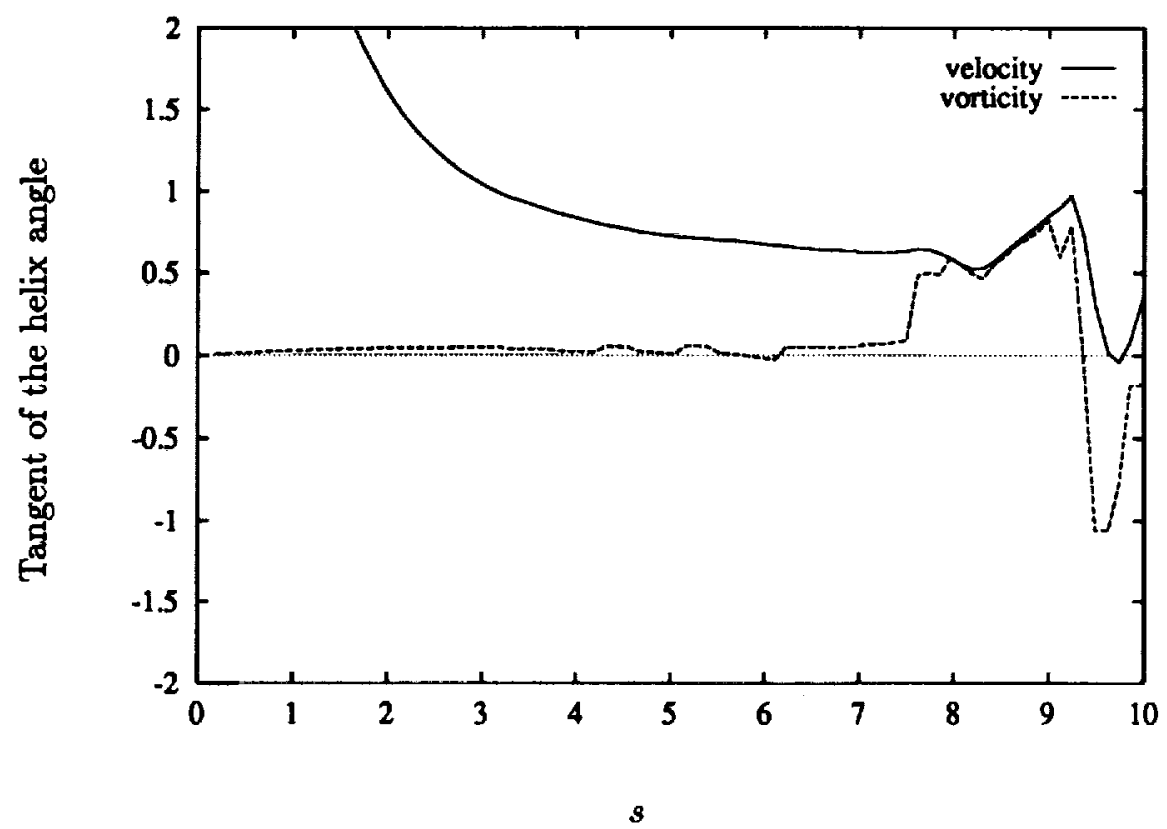

Figure 11. Axial distribution of the tangents of the helix angles of velocity and vorticity at the radius of maximum azimuthal velocity at time $t=28.5$.

stages in the evolution of an axisymmetric vortex beginning to undergo vortex breakdown.

Figure 10 shows contours of enstrophy. The maximum enstrophy is at the center of the vortex and has diminished to about half its maximum value at the topology change. Where the azimuthal vorticity is at its maximum, the enstrophy has decreased to approximately one fifth of its maximum value.

The ratio of the tangents of the helix angles of the velocity and vorticity vectors in region $I$ at the edge of the vortex core is much larger than one (see Figure 11). This indicates that the streamwise rib vortex, which is nearly axisymmetric in region I, has a structure which satisfies a necessary condition for an inviscid, axisymmetric vortex to undergo vortex breakdown. The primarily axially directed vorticity vector is locally turned into the negative azimuthal direction, and the corresponding induced velocity results in a deceleration of the axial flow as detailed in Brown and Lopez (1990). The temporal and spatial developments, particularly of azimuthal vorticity, are similar to those found by Brown and Lopez (1990) for an isolated axisymmetric vortex in the early stages of vortex breakdown. Contours of the azimuthal vorticity from that study are presented in Figure 12. Initially, the azimuthal vorticity close to the axis is positive with little axial variation. The inflow profile is time independent. Slow radial diffusion, due to the finite Reynolds number of this flow, results in a slight broadening of the vortex core and a slight slowing of the axial flow. Off the axis, the initially axially directed vorticity vector has been 


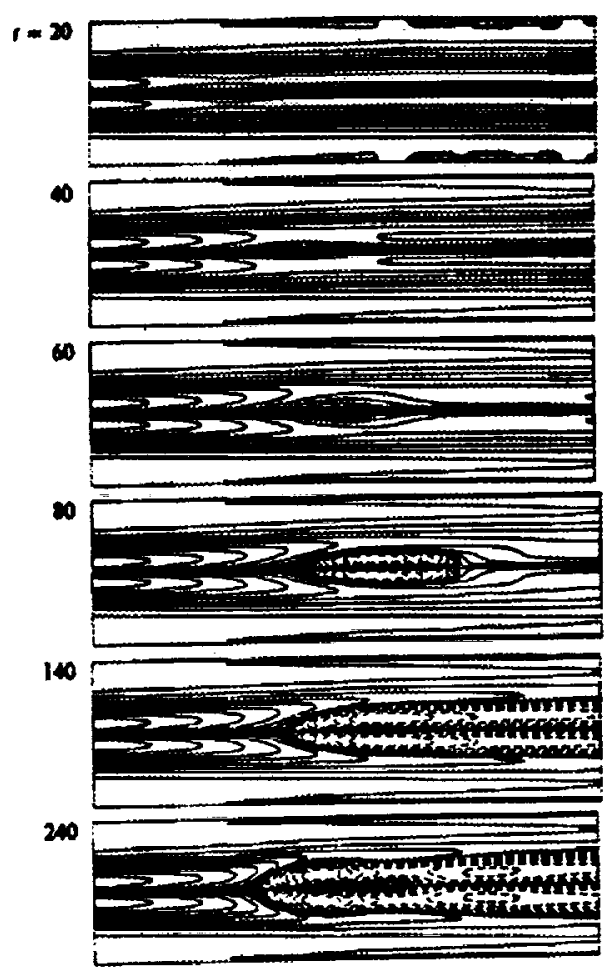

FIGURE 12. Azimuthal vorticity contours for an isolated axisymmetric vortex (reproduced from Brown and Lopez 1990).

turned into the negative azimuthal direction. This is enhanced locally by the nonlinear feed-back mechanism detailed in Brown and Lopez (1990). By time $t=80$, a local concentration of negative azimuthal vorticity has developed. This is advected downstream by the mean axial flow at the same time as it is growing in intensity through the non-linear process. By time $t=240$, the negative azimuthal vorticity is large enough to induce a reversed axial flow and begins to propagate upstream under its own induced velocity. The major difference between the evolution of the rib vortex and the isolated axisymmetric vortex is the lack of a significant positive azimuthal component of vorticity in the rib vortex. Nevertheless, in both cases, the ratio of helix angles is greater than one, the region of negative azimuthal vorticity initially travels downstream, and there is an associated deceleration of the axial flow, underlining the similarities between the two cases.

\section{Vortex-vortex interactions in region III}

It is clear from the discussion in the previous section that the rib vortex, at the times considered, is in the early stages of axisymmetric vortex breakdown. However, the most striking aspect of the structural development of the rib vortex is not 
(a)

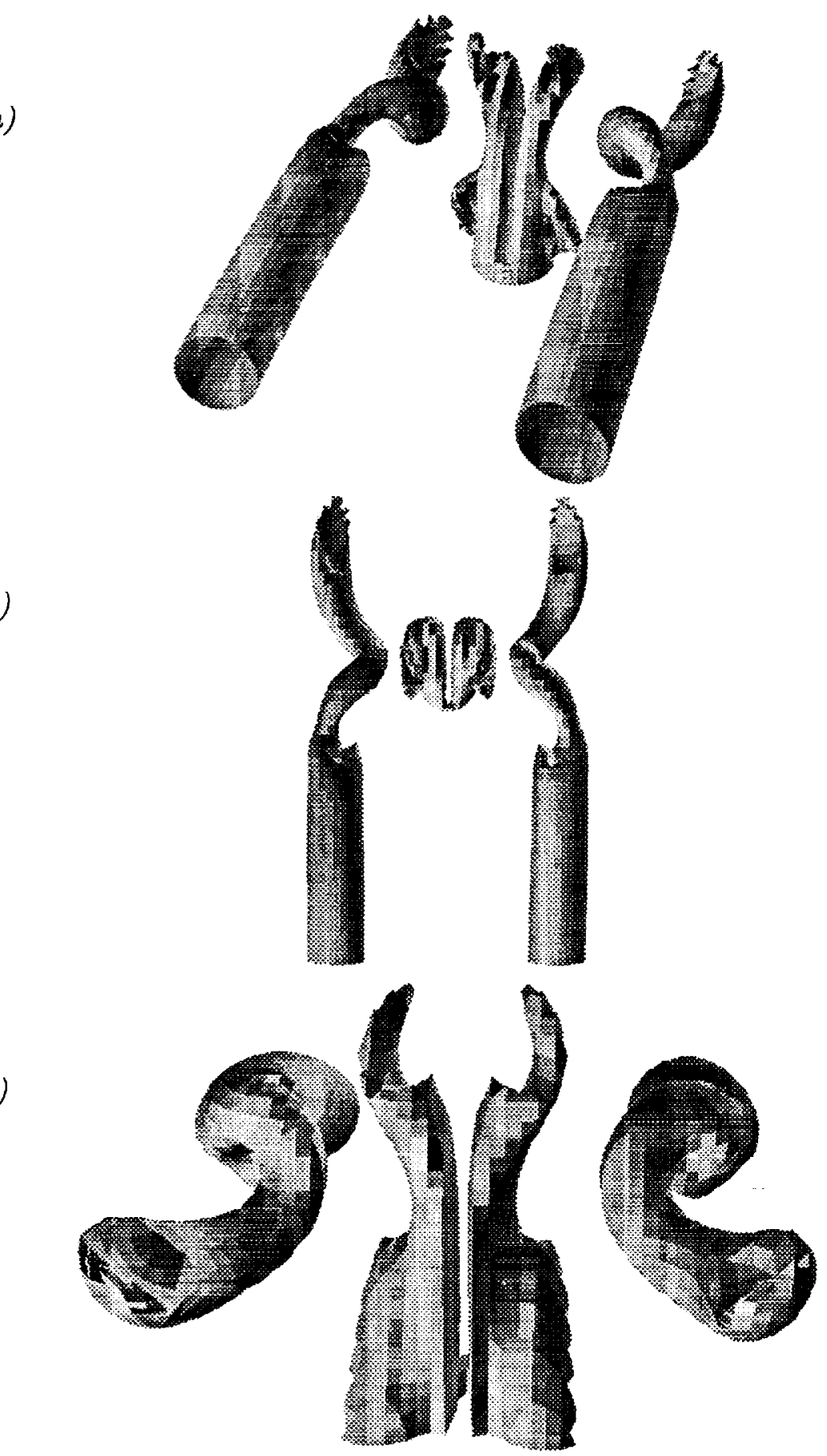

(b)

(c)

FIGURE 13. Counter rotating streamwise rib vortices visualized by an enstrophy isosurface of level 3.2: (a) perspective view looking downstream, (b) view from top, and (c) view looking upstream. 
directly associated with the vortex breakdown process occurring in regions I and II. At the latest time considered, $t=28.5$, the vortex has developed a very distinctive spiral in region III. Figure 13 gives three views of the structure as visualized by an isosurface of the enstrophy. Shown are a pair of counter rotating rib vortices and some concentrated vortical flow between them.

Due to the spanwise periodicity in the flow, the rib vortices are present as an array of vortices with alternating senses of rotation across the mixing layer. This arrangement results in their drawing vortical fluid from within the spanwise roller region to between the rib vortices, whose induced velocity in the plane between them is directed vertically outward from the spanwise roller region. Bernal and Roshko (1986) have demonstrated this effect visually using spanwise laser-light sheets illuminating fluorescent dye which has been introduced into the flow. The observed mushroom-like structures indicate the locations of the rib vortices.

At time $t=22.3$, the rib vortex pairs have not yet drawn much vortical fluid out between them. By $t=25.3$, the vortical flow between the rib vortex pair has begun to collapse into a vortex loop, following the scenario depicted by Corcos (1988). The enstrophy associated with this vortex loop is diffuse and of elliptic cross section. By $t=28.5$, the vortex loop has been stretched by the induced velocity from the streamwise rib vortex pair. In the neighborhood of the rib pair, the vortex loop has been stretched and intensified into a vortex pair, which is connected by a loop. Three different views of these vortices are given in Figure 14, where vortex lines passing through the center of the vortices have been used as well as an isosurface of enstrophy. These figures clearly show the spiralling of the rib vortex pair. Note that the sense of the spiral is opposite to that of the sense of rotation of the vortex. The spiralling of the rib vortex pair occurs at the axial location closest to the intensified vortex loop, suggesting that the spiralling is due to a vortex-vortex interaction between the rib vortex and a leg of the vortex loop.

The ratio of enstrophy in the rib vortices to that in the spanwise rollers needed for the rib vortices to draw up vortical flow and intensify it by vortex stretching into a counter-rotating pair between a pair of streamwise rib vortices remains to be determined.

\section{Conclusions}

From this study of the streamwise rib vortices in a three- dimensional plane mixing layer, it is evident that these vortices evolve from an essentially axisymmetric state, well-described as a Burger's vortex. The vorticity vector, initially aligned with the axial direction, is susceptible to being turned into the negative azimuthal direction by the swirling flow. Brown and Lopez (1990) have shown that this inviscid process is possible when the tangent of the helix angle of the velocity vector is larger than that of the vorticity vector, as is the case for the rib vortices investigated. This structure is typical of the rib vortex downstream of the mid-braid plane and upstream of the topology change. Evidence has been found that it begins to undergo vortex breakdown. This is indicated by the change in the local topology, the development of a negative azimuthal component of vorticity, a deceleration of 
(a)
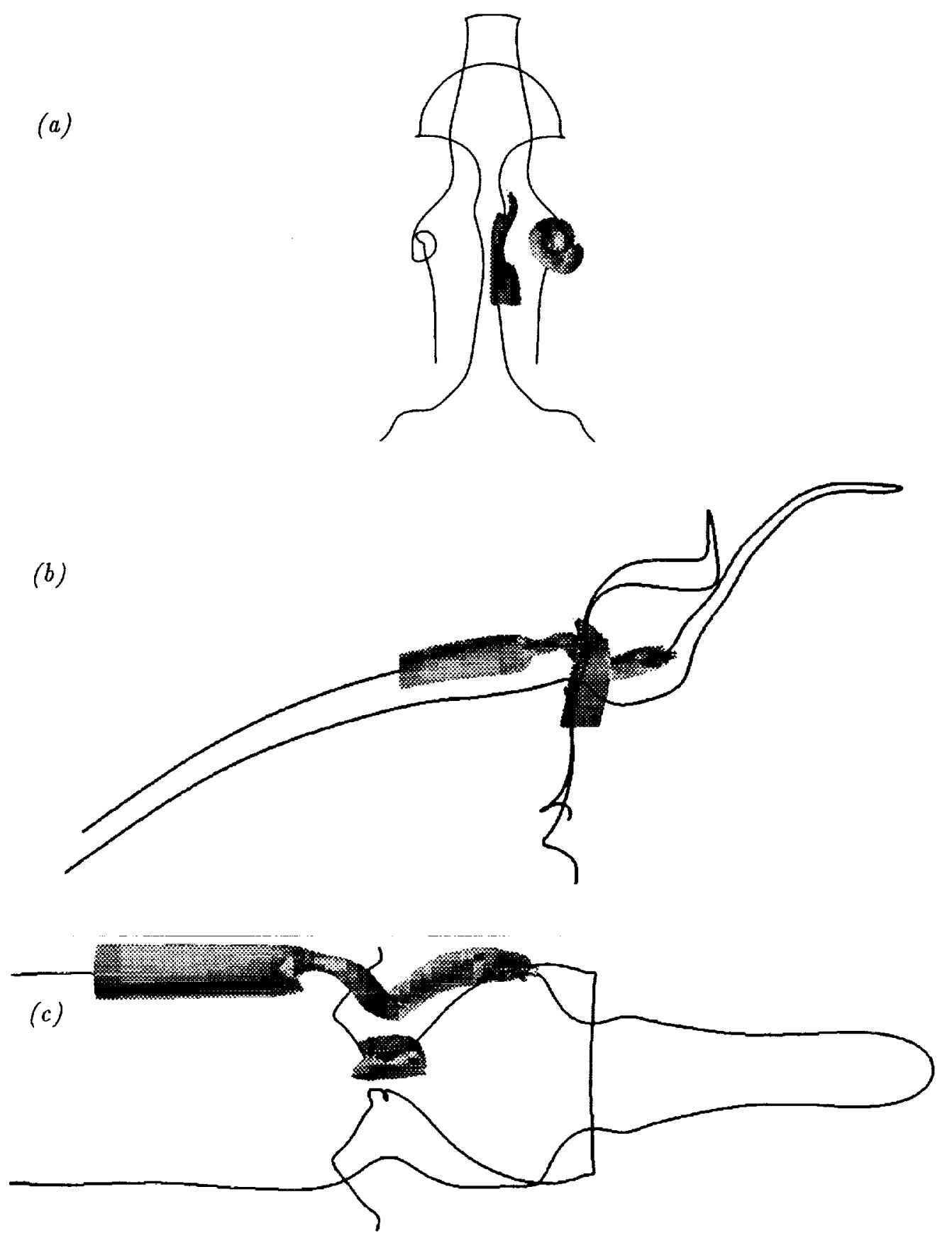

$=$

FiguRE 14. Counter rotating streamwise rib vortex pair and intensified vortex loop visualized by an enstrophy isosurface of level 3.2 and vortex lines: $(a)$ view looking upstream, (b) spanwise view, and (c) top view. 
the axial flow along the vortex axis, and a departure from axial symmetry downstream of the topology change. This provides a natural division of the structure of the rib vortex into two distinct regions on the vortex axis: where the topology is stable focus/stretching and where it is unstable focus/contracting. At the later times considered, there is a third structurally distinct region downstream of the two described above. In this region, the spiralling of the vortex core is due to a vortex-vortex interaction between the streamwise rib vortex and the vortical flow in the main spanwise roller region which has been drawn up and stretched by two counter- rotating adjacent rib vortices.

Although vortex breakdown is beginning to take place at the times considered, it does not appear to be having much effect on the rest of the flow. There is no sudden core expansion, recirculation zone, or intense mixing, phenomena normally associated with large-scale vortex breakdown flows. Certainly the vortex-vortex interaction dominates the flowfield in region III. However, vortex breakdown in small-scale flows may play a role in defining a length scale for vortical structures. In the rib vortex, the region in which the enstrophy is largest compared with the mean flow is bounded by the topology change, a change which can be attributed to vortex breakdown in the rib vortex. In small scale flows, viscous dissipation and interactions with other similar structures have previously been regarded as the two mechanisms whereby a finite length is imposed on a vortex. Vortex breakdown, essentially an inviscid phenomenon, should be considered the third.

It would be interesting to follow the evolution of this flow further in time to determine whether the vortex breakdown develops further into a well defined recirculation zone or if a further pairing of the main rollers occurs before this eventuates.

\section{Acknowledgements}

We would like to thank Mike Rogers, Bob Moser, Julio Soria, Brian Cantwell and Rolf Sondergaard for discussions during the 1992 CTR Summer Program and Bruce Fairlie for his comments.

\section{REFERENCES}

Bernat, L. P. \& Roshкo, A. 1986 Streamwise vortex structure in plane mixing layers. J. Fluid Mech. 170, 499-525.

Brown, G. L. \& Lopez, J. M. 1990 Axisymmetric vortex breakdown. Part 2. Physical Mechanisms. J. Fluid Mech. 221, 553-576.

Chen, J. H., Chong, M. S., Soria, J., Sondergaard, R., Perry, A. E., Rogers, M., Moser, R. \& Cantwell, B. J. 1990 A study of the topology of dissipating motions in direct numerical simulations of time-developing compressible and incompressible mixing layers. Proceedings of the Center for Turbulence Research Summer Program 1990. Stanford Univ./NASA Ames.

Chong, M. S., Perry, A. E. \& Cantwell, B. J. 1990 A general classification of three-dimensional flow fields. Phys. Fluids A. 2, 765-777. 
Concos, G. M. 1988 The role of cartoons in turbulence. In Perspectives in Fluid Mechanics (ed. D. E. Coles) Lecture Notes in Physics, vol. 320, pp. 48-65. (Springer).

DREsselhaUs, E. \& TABOR, M. 1991 The kinematics of stretching and alignment of material elements in general flow fields. J. Fluid Mech. 236, 415-444.

Jiménez, J. 1992 Kinematic alignment effects in turbulent flows. Phys. Fluids A. 4, 652-654.

Kelso, R. $1992 \mathrm{PhD}$ Thesis, University of Melbourne, Australia.

Moser, R. \& Rogers, M. 1992 The three-dimensional evolution of a plane mixing layer: pairing and transition to turbulence. J. Fluid Mech., submitted for publication.

Orszag, S. A. 1991 Otto Laporte lecture, $44^{\text {th }}$ meeting APSDFD, Scottsdale AZ.

Perry, A. E. \& FAIrlie, B. D. 1974 Critical points in flow patterns. Adv. Geophys.. 18, 299-315.

SHE, Z.-S., JACKSON, E. \& ORSzAG, S. A. 1990 Intermittent vortex structures in homogeneous isotropic turbulence. Nature. 344, 226-228.

Yates, L. A. \& Chapman, G. T. 1992 Streamlines, vorticity lines, and vortices around three-dimensional bodies. AIAA Journal. 30, 1819-1826. 